\section{Bias in the COVID-19 era}

\section{DOI: $10.7861 /$ clinmed.Let.21.1.4}

Editor - We thank Kelly et al for an informative and useful article. ${ }^{1}$ There is only a short mention of clinical biases, and I believe that this point needs further attention. Cognitive biases are omnipresent in medicine. They can affect reasoning and result in adverse events. ${ }^{2} \mathrm{~A}$ few types of biases exist here. One was confirmation bias which is the analysis of information in a particular way to confirm and strengthen original suspicions or hypotheses. This was most probably in play on the patient's first presentation where the diagnosis of 'mild COVID-19' was entertained. There is also clear availability bias, this is an intrinsic human tendency where one assesses the likelihood of an event by how often other examples come to one's mind, thus creating mental shortcuts. Anchoring bias was also at play, this is where one settles on a diagnosis based on a few important features. It is difficult to know if affective bias was present, which is the tendency to convince oneself what one wants to be true. It is also easy to perform a retrospective analysis of any case such as this one and this is by no means a criticism of the initial treatment, but COVID-19 mimics are increasingly being described. ${ }^{3}$ Good clinical acumen and an understanding of cognitive biases (which are not taught at medical school and only come from continuous adult learning and reflective practice) will make sure that patients continue to receive good medical care.

AVINASH AUJAYEB

Consultant in respiratory and acute medicine, Northumbria Healthcare NHS Foundation Trust, Cramlington, UK

\section{References}

1 Kelly S, Waters L, Cevik M et al. Pneumocystis pneumonia, a COVID-19 mimic, reminds us of the importance of HIV testing in COVID-19. Clin Med 2020;20:590-2.

2 O'Sullivan ED, Schofield SJ. Cognitive bias in clinical medicine. J $R$ Coll Physicians Edinb 2018:48:225-32.

3 Hanfi SH, Lalani TK, Saghir A et al. COVID-19 and its mimics: what the radiologist needs to know. J Thorac Imaging 2020 [Epub ahead of print].

\section{General management approach to common vasculitides}

\section{DOI: $10.7861 /$ clinmed.Let.21.1.5}

Editor - We recently read the article, 'An update on the general management approach to common vasculitides' by Hng, Zhao and Moots. ${ }^{1}$

We would like to suggest refinements of the description of dermatological manifestations in a patient with probable vasculitides as outlined their Table 2 and Fig 2, as we are concerned that comments like 'new persistent rash' might be a little too generalised and non-specific.

This issue is important to be addressed since there have been significant updates of the classification of vasculitis and dermatologists have strived to define lesions of vasculitis in patients with greater accuracy, and the Chapel Hill Consensus of 2018 reflects those efforts. ${ }^{2}$

In very simple terms, skin examination findings in vasculitis relate to vessel size. Small vessel vasculitis causes a palpable petechial rash. Medium vessel involvement on the other hand leads to livedo reticularis (a lace like discoloration of skin), ulceration, nodules or macules; while large vessel involvement leads to skin necrosis and ulceration.

We hope our comments are useful and add to the educational aspects of the paper.

SHRIYA KACHRU

Clinical attachee in general internal medicine, Queen Alexandra Hospital, Cosham, UK

ALEXA SHIPMAN

Consultant dermatologist, Queen Alexandra Hospital, Cosham, UK

\section{References}

1 Hng M, Zhao SS, Moots RJ. An update on the general management approach to common vasculitides. Clin Med 2020;20:572-9.

2 Sunderkötter $\mathrm{CH}$, Zelger B, Chen KR et al. Nomenclature of Cutaneous Vasculitis: Dermatologic Addendum to the 2012 Revised International Chapel Hill Consensus Conference Nomenclature of Vasculitides. Arthritis Rheumatol 2018;70:171-84.

\section{HIV testing during the COVID-19 pandemic}

\section{DOI: 10.7861/clinmed.Let.21.1.6}

Editor - We support the recommendations made in the recent report by Kelly et al for increased awareness of importance of HIV testing during the COVID-19 pandemic. ${ }^{1}$

During the UK's first wave, our hospital trust in north west London experienced a high burden of COVID-19 patients, with clinical staff quickly becoming familiar with the clinical and radiological features of COVID-19. Studies that have compared pooled nasopharyngeal aspirate results with cross-sectional imaging indicate that the sensitivity of reverse transcriptase polymerase chain reaction (RT-PCR) for SARS-CoV-2 may be very poor. ${ }^{2}$ As a result, a significant proportion of COVID-19 patients are diagnosed on the basis of typical clinical and radiological features, despite negative tests. ${ }^{3}$

Between March and June 2020, three patients were admitted with signs and symptoms consistent with COVID-19 (respiratory failure and bilateral infiltrates on chest imaging) but were COVID-19 RT-PCR negative. These patients were subsequently diagnosed with advanced HIV (mean CD 4 count $62 \times 10^{6} / \mathrm{L}$ (range $\left.12-108 \times 10^{6} / \mathrm{L}\right)$ ) and Pneumocystis jirovecii pneumonia (PCP). Due to the initial presumed clinical diagnosis of COVID-19 there was a delay in testing for HIV and diagnosing and treating PCP.

PCP and COVID-19 are difficult to distinguish on clinical grounds, with shared symptoms including fever, dyspnoea, dry cough and fatigue; although, in COVID-19 the onset is more acute. ${ }^{4,5}$ The similar radiological findings include bilateral infiltrates with COVID-19 typically involving the lung peripheries whereas PCP is often peripherally sparing. ${ }^{5}$

Our experience highlights the importance of maintaining diagnostic vigilance when pursuing a diagnosis of COVID-19. We strongly advocate HIV testing all individuals presenting with acute respiratory illness, including those with 'typical' features of COVID-19 as per UK national guidelines. ${ }^{6}$

SARAH ANN FILSON

Infectious diseases and microbiology registrar, London North West University Healthcare NHS Trust, London, UK 
ANIKA RAHIM

Internal medical trainee, Royal Free London NHS Foundation Trust,

London, UK

VICTORIA PARRIS

Consultant in infectious diseases and acute medicine, London North West University Healthcare NHS Trust, London, UK

ALASTAIR McGREGOR

Consultant in infectious diseases and microbiology, London North West University Healthcare NHS Trust, London, UK

PADMASAYEE PAPINENI

Consultant in infectious diseases and acute medicine, London North West University Healthcare NHS Trust, London, UK

\section{References}

1 Kelly S, Waters L, Cevik M et al. Pneumocystis pneumonia, a COVID-19 mimic, reminds us of the importance of HIV testing in COVID-19. Clin Med 2020;20:590-2.

2 Ai T, Yang Z, Hou H et al. Correlation of chest CT and RT-PCR testing for coronavirus disease 2019 (COVID-19) in China: A report of 1014 cases. Radiology 2020;296:E32-40.

3 Tavare AN, Braddy A, Brill S et al. Managing high clinical suspicion COVID-19 inpatients with negative RT-PCR: a pragmatic and limited role for thoracic CT. Thorax 2020;75:537-8.

4 Choy CY, Wong CS. It's not all about COVID-19: pneumocystis pneumonia in the era of a respiratory outbreak. J Int AIDS Soc 2020;23:e25533.

5 Coleman JJ, Manavi K, Marson EJ et al. COVID-19: to be or not to be; that is the diagnostic question. Postgrad Med J 2020;96:392-8.

6 British HIV Association, British Association for Sexual Health and HIV, British Infection Association. British HIV Association / British Association for Sexual Health and HIV / British Infection Association adult HIV testing guidelines 2020. BHIVA, 2020. www. bhiva.org/file/5f68c0dd7aefb/HIV-testing-guidelines-2020.pdf [Accessed 23 November 2020].

\section{Vitamin D}

\section{DOI: $10.7861 /$ clinmed.Let.21.1.7}

Editor - In their recent article, Griffin et al suggest that all adults should receive 800-1,000 IU vitamin D daily supplementation, perhaps to reduce the impact of the COVID-19 pandemic. ${ }^{1}$ They point to an observed association between low serum 25-hydroxyvitamin $\mathrm{D}(25(\mathrm{OH}) \mathrm{D})$ concentration and COVID-19 seropositivity in a recent study involving two of the current article's authors. ${ }^{2}$ In addition, they highlight a small, open label, pilot study from Spain $(n=76)$, which suggested a lower rate of intensive care unit admission with vitamin D supplementation compared with placebo (baseline and on-treatment 25(OH)D concentrations not reported). ${ }^{3}$

There is a familiar tale of vitamin $D$ that has been repeated over the last few decades. Observational studies associate low serum $25(\mathrm{OH}) \mathrm{D}$ with numerous adverse health outcomes. ${ }^{4,5}$ Yet despite tens of thousands of people being randomised into studies, the evidence for any health benefit from vitamin $D$ supplementation still evades us. ${ }^{6-7}$ The reason for the association of low 25(OH)D and adverse outcomes is multifactorial; in part related to people with poorer health status getting less sunlight exposure and having reduced dietary intake; in part due to the negative acute phase response of serum $25(\mathrm{OH}) \mathrm{D}$ (ie it is lowered in times of bodily inflammation). ${ }^{8}$
Through the majority of their article, Griffin et al consider the serum concentration at which $25(\mathrm{OH}) \mathrm{D}$ would justify supplementation. The argument for a higher threshold $(50 \mathrm{nmol} / \mathrm{L}$ ) being mainly supported by the observation that elevating serum $25(\mathrm{OH}) \mathrm{D}$ concentration suppresses parathyroid hormone release. But does this lead to any tangible health benefits?

There is some evidence that only $25(\mathrm{OH}) \mathrm{D}$ concentrations of $10 \mathrm{nmol} / \mathrm{L}$ or below create significant biochemical disorders, such as hypocalcaemia. ${ }^{9}$ Improved bone health has been examined as a potential major benefit of vitamin D supplementation. A study that randomised 2,578 people aged over 70 years (mean age 80 ) compared vitamin D supplementation with placebo over a median follow-up of 3.5 years. ${ }^{10}$ Despite higher serum $25(\mathrm{OH}) \mathrm{D}$ concentrations achieved with supplementation $(60 \mathrm{nmol} / \mathrm{L}$ vs $23 \mathrm{nmol} / \mathrm{L}$ ), no reduction in fracture rate was detected. These data do not support accepting $<50 \mathrm{nmol} / \mathrm{L}$ as the threshold for $25(\mathrm{OH}) \mathrm{D}$ deficiency.

In any case, Griffin et al are not advocating a treat-to-target approach, instead a blanket supplementation for all adults. Although the risk of harm may be small, adverse effects would include some gastrointestinal symptoms and occasional cases of hypercalcaemia. ${ }^{7}$ Additional tablets would contribute to the growing burden of polypharmacy for many people. But perhaps most importantly, what would be the opportunity cost? Instead of using resources putting unwanted and unopened boxes of pills in every home in Britain and Ireland, we could be investing in shared decision-making processes to encourage health improvement for individuals under our care. Promoting non-pharmacological approaches, including smoking cessation and exercising outdoors, would have a far greater impact on our nations' well-being.

HENRY ] WOODFORD Consultant geriatrician, Northumbria Healthcare NHS Foundation Trust, UK

\section{References}

1 Griffin G, Hewison M, Hopkin J et al. Preventing vitamin D deficiency during the COVID-19 pandemic: UK definitions of vitamin D sufficiency and recommended supplement dose are set too low. Clin Med 2021;21:e48-51.

2 Faniyi AA, Lugg ST, Faustini SE et al. Vitamin D status and seroconversion for COVID-19 in UK healthcare workers who isolated for COVID-19 like symptoms during the 2020 pandemic. medRxiv 2020.10.05.20206706.

3 Castillo ME, Entrenas Costa LM, Vaquero Barrios JM et al. Effect of calcifediol treatment and best available therapy versus best available therapy on intensive care unit admission and mortality among patients hospitalized for COVID-19: A pilot randomized clinical study. J Steroid Biochem Mol Biol 2020;203:105751.

4 Schottker B, Jorde R, Peasey AS et al. Vitamin D and mortality: meta-analysis of individual participant data from a large consortium of cohort studies from Europe and the United States. BMJ 2014;348:93656.

5 Bolland MJ, Avenell A, Grey A. Should adults take vitamin D supplements to prevent disease? BMJ 2016;355:16201.

6 Theodoratou E, Tzoulaki I, Zgaga L et al. Vitamin D and multiple health outcomes: umbrella review of systematic reviews and meta-analyses of observational studies and randomised trials. BMJ 2014:348:g2035.

7 Avenell A, Mak JCS, O'Connell D. Vitamin D and vitamin D analogues for preventing fractures in post-menopausal women and older men. Cochrane Database Syst Rev 2014;2014:CD000227. 